\title{
Flap reconstruction of soft tissue defect after resecting a huge hemangioma of the nose
}

\author{
Joonho Lim, \\ Jeongseok Oh, \\ Seokchan Eun \\ Department of Plastic and \\ Reconstructive Surgery, Seoul National \\ University Bundang Hospital, Seoul \\ National University College of Medicine, \\ Seongnam, Korea
}

\begin{abstract}
Hemangioma is a benign vascular tumor that grows by endothelial cell hyperplasia. It occurs most frequently in the head and neck region. Nose reconstruction is tricky because of its unique threedimensional structure and different tissue components. We report a case of successful reconstruction of near-total nose defect using the paramedian forehead flap combined with a nasolabial flap, immediately after excision of nasal hemangioma. A 49-year-old male patient was presented with a huge mass at the nose. Preoperative magnetic resonance imaging showed prominent vascular channels extending to the forehead and cheek. Complete resection of the mass was performed, which resulted in an eccentric defect. The right paramedian forehead flap and the left nasolabial flap were designed and transferred to the defect. Flap division was performed 1 month later. The patient is satisfied with the overall appearance and did not develop any functional deficit.
\end{abstract}

Keywords: Hemangioma / Nasal surgical procedures / Nose neoplasms / Surgical flaps

\section{INTRODUCTION}

The nose is a major landmark of the face that defines the overall appearance of a face. Due to its prominent location, even minor disfigurements can be discernible. Several tissue components, including skin, fibrofatty soft tissue, cartilaginous framework, nasal bone, and intranasal lining, form the unique structure.

Historically, nose reconstruction has been a foundation of reconstructive plastic surgery [1]. The optimal method of nose reconstruction remains controversial regardless of the extensive literature. Ideal reconstruction of nose defects should recreate both aesthetic contour and function. Factors such as size, location, and depth should be assessed to ensure optimal reconstruction.

First described a thousand years ago, the forehead flap has

\footnotetext{
Correspondence: Seokchan Eun

Department of Plastic and Reconstructive Surgery, Seoul National University Bundang Hospital, Seoul National University College of Medicine, 82 Gumi-ro 173beon-gil, Bundang-gu, Seongnam 13620, Korea

E-mail: seokchaneun@gmail.com

Received November 8, 2019 / Revised January 10, 2020 / Accepted January 28, 2020
}

been refined in numerous modifications. It is considered to be the workhorse flap for large nasal defects because it provides similar skin characteristics and structural support with high reliability [2]. We present a successful nose reconstruction combining a paramedian forehead flap and nasolabial flap after the resection of a huge hemangioma.

\section{CASE REPORT}

A 49-year-old male patient presented with a nasal mass of $8 \times 5$ $\mathrm{cm}$. The lesion was red to brown in color, irregular in shape and surface (Fig. 1). He had two partial resections of the lesion about 25 years ago, but pathology reports and other surgical details were not obtainable. Since then, he visited the emergency room with bleeding from the recurring lesion. The bleeding stopped, but he was referred to the outpatient clinic for the lesion. The medical history was otherwise unremarkable.

The paranasal sinus computed tomography revealed an 8.1 $\mathrm{cm}$-sized heterogeneously enhancing mass with rich vascularity at the nasal tip, suggesting vascular malformation. The naso- 


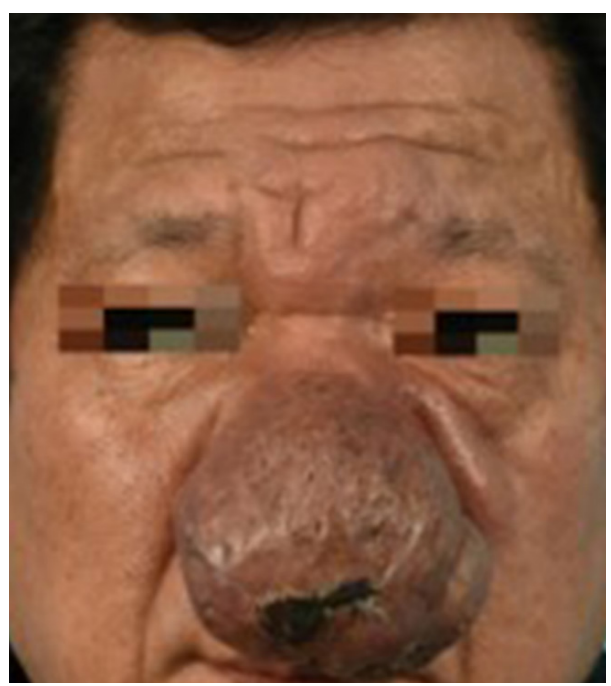

Fig. 1. Preoperative clinical photograph. A male patient presented with a nasal mass of $8 \times 5 \mathrm{~cm}$.

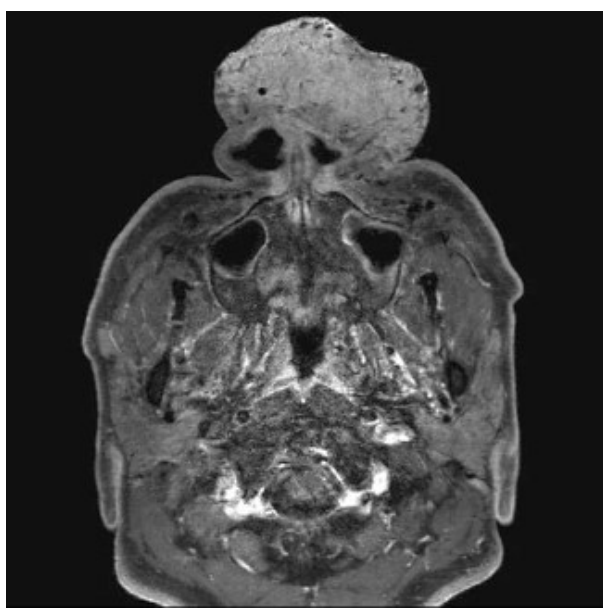

Fig. 2. The nasopharyngeal magnetic resonance imaging revealed an $8.0 \times 3.5 \times 6.7 \mathrm{~cm}$-sized hypervascular mass at the nose with multiple vascular channels to surrounding tissues, suggesting a non-involuting congenital hemangioma.

pharyngeal magnetic resonance imaging revealed an $8.0 \times$ $3.5 \times 6.7 \mathrm{~cm}$-sized hypervascular mass at the nose with multiple vascular channels to surrounding tissues, suggesting a non-involuting congenital hemangioma (Fig. 2). The channels were located at bilateral forehead, glabella, nasal dorsum, preantral cheek, and chin. The channels were also evident underneath the zygomaticus major muscles.

The mass was carefully resected under general anesthesia. The resection resulted in an exposure of bilateral upper lateral and lower lateral cartilages (Fig. 3). The defect involved the whole of columella, nasal tip, and left ala. The defect also affected a part of the nasal dorsum and left cheek. Two flaps, the paramedian forehead flap based on the right supratrochlear artery and the

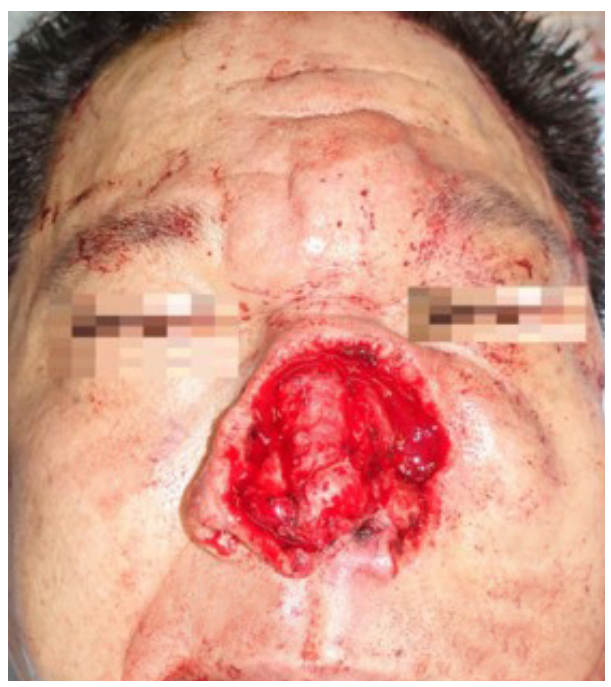

Fig. 3. Intraoperative clinical photograph. The resection resulted in an exposure of bilateral upper lateral and lower lateral cartilages. The defect involved the whole of columella, nasal tip, and left ala. The defect also affected a part of the nasal dorsum and left cheek.

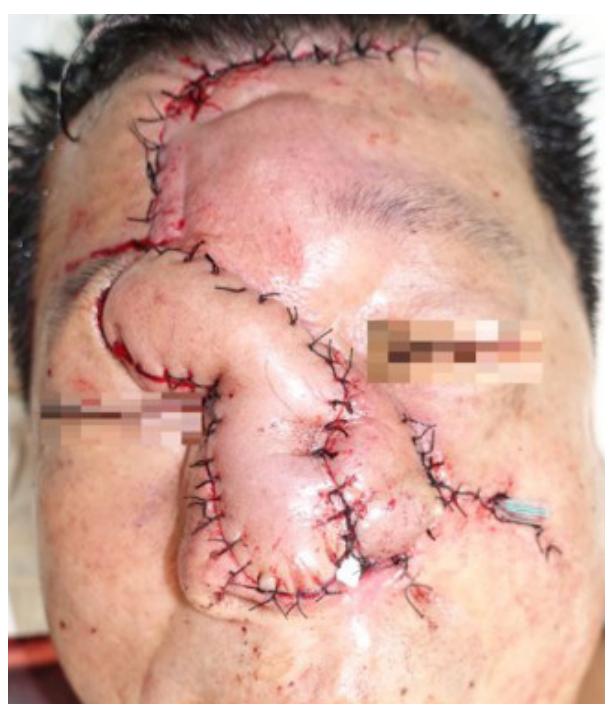

Fig. 4. Immediate postoperative clinical photograph. The paramedian forehead flap based on the right supratrochlear artery and the nasolabial rotation flap from the left side were utilized to cover the defect.

nasolabial rotation flap from the left side, were utilized to cover the defect (Fig. 4). The two donor sites were closed primarily without remarkable disfigurement. The forehead flap was divided 4 weeks after the first operation. Minor surgical refinements were also made with the flap division. Before closing the proximal wounds, excessive soft tissue around the split-site was carefully removed to achieve a good postoperative shape. A wedge resection of the left alar base area was done to correct the superiorly displaced alar. The patient showed good aesthetic results, without any complication or recurrence for 10 months 


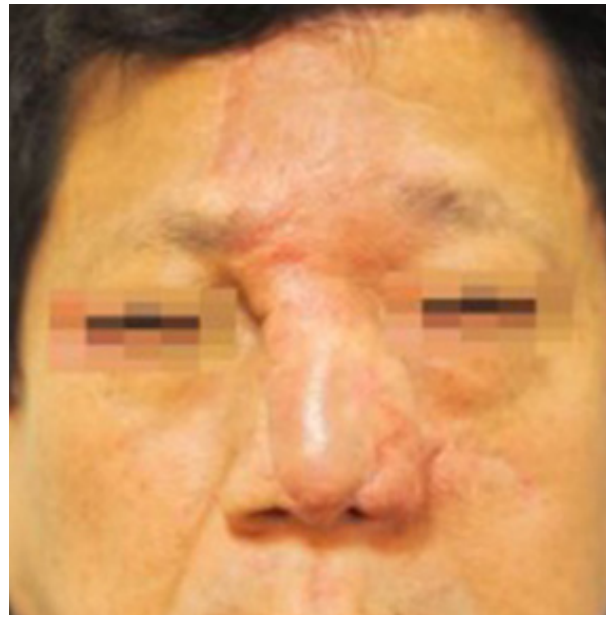

Fig. 5. Postoperative clinical photograph at 10 months. The patient showed good aesthetic results, without any complication or recurrence.

after the surgery (Fig. 5). The surgical pathology confirmed a non-involuting congenital hemangioma with moderate fibrosis.

\section{DISCUSSION}

According to the International Society for the Study of Vascular Anomalies, vascular anomalies are split into two main entities: vascular tumors and vascular malformations [1,2]. Hemangioma is a benign vascular tumor that grows by endothelial cell hyperplasia. Histology of hemangioma shows an increased number of vascular structures lined by a monolayer of endothelial cells. Such structures are easily recognizable because they contain red blood cells or transudate. Hemangioma occurs most frequently in head and neck region, followed by the trunk and the extremities. About $60 \%$ of hemangiomas occur in head and neck region.

Hemangioma is divided into two groups: infantile hemangioma and congenital hemangioma. Congenital hemangioma clinically presents as fully developed lesions at birth. It rapidly involutes during the first year of life or may never show involution.

If a hemangioma does not go away or causes other medical problems, it requires treatment. The treatment may include medications, laser therapy, or surgical excision. Beta-blocker drugs and corticosteroids are possible choices for medical therapy [3]. Surgical excision is considered if a hemangioma hinders function, development, or cosmesis. Non-involuting congenital hemangiomas often necessitate surgical extirpation. The hemangioma of this case was extremely large that the benefit of surgical resection was obvious.

Defects of the nose are caused by various situations including animal bite, trauma, and tumor resection [4]. Numerous surgical methods and concepts for nasal reconstruction have been suggested, but there is no consensus on how to establish a surgical strategy for each case. The three-layer approach based on nose anatomy is the kernel of nasal defect analysis for reconstruction. The nose can be divided into three layers: an external layer of covering skin, a middle layer of osteocartilaginous support framework, and an internal layer of lining mucosal membrane. The anatomical layer-by-layer repair of the defect should be achieved as much as possible.

The subunit principle also had a high impact on nasal analysis before the reconstruction. Burget and Menick introduced the principle [5]. Subunits are designated as slightly convex and concave surfaces separated by ridges and valleys, including the dorsum, tip, ala, sidewalls, and soft triangles. Not only did they cover the defect, but they substituted the whole subunit in which the defect affected. This strategy causes postoperative scars to mimic the ridges and valleys of the nasal skin. On the contrary, this principle always involves the removal of healthy neighboring tissue and therefore requires a flexible application.

The forehead flap, first described in India approximately a thousand years ago, has become the ultimate tool in the reconstruction of extensive nasal defects after a series of improvements [6]. Originally, the midline forehead flap was preferred over the paramedian forehead flap. However, the paramedian forehead flap was favored due to its reliability, manageability, and excellent cosmesis in restoring the large nasal defect. The supratrochlear artery, the main vascular pedicle of the flap, has a relatively constant anatomy of $1.7-2.2 \mathrm{~cm}$ lateral to the midline when exiting the orbit [7]. A two-staged paramedian forehead flap with periosteal cuff was applied in this case. The cuff helped the secure fixation of the flap. The aesthetic result was fairly good in this case, but also showed the minor limitations of the forehead flap. Consuming the tissue of the left forehead resulted in the raised height of the left eyebrow. Also, there were some extra tissue and slight irregularities on the right eyebrow and the right side of the nasal radix, which was around the former site of the flap pedicle. Such deformities can be improved via secondary minor revisions at the outpatient clinic.

The nasolabial flap is a useful and ideal modality mainly used for alar defects. Its proximity to the defect not only makes color and texture perfectly match but also makes it easy to cover the defect. Besides its proximity and the natural cheek laxity, subcutaneous dissection into the cheek provides additional laxity, facilitating closure with minimizing distortion of the surrounding tissue. Thoughtful design can hide the postoperative donor in the nasolabial fold.

This case report highlights two implications. Firstly, an accu- 
rate diagnosis is critical. It was unlikely, but a doctor could have been misdiagnosed the patient as rhinophyma due to its location. Rhinophyma is characterized by slowly progressing strawberry nodules on the nasal skin and severely disrupts the nasal contour [8]. It rarely occurs in Asians, but rhinophyma should be ruled out in this case. As rhinophyma is a lesion caused by hypertrophy of sebaceous glands, initial treatment includes dermabrasion and laser therapy in an outpatient clinic [9]. These treatments could have provoked accidental bleeding from ruptured hemangioma.

Secondly, combining two classic workhorse flaps can produce satisfying results in nasal reconstruction. On the current trend of the reconstructive elevator, even a moderate defect tempts the surgeon to transfer a free flap. Nevertheless, it may be better to overcome traditionally, and this is the case. Considering that the patient did not seek any treatment for the remarkably large mass at the nose, it might be harder to expect prudent compliance, which is an influential factor for free tissue transfer survival.

\section{NOTES}

\section{Conflict of interest}

No potential conflict of interest relevant to this article was reported.

\section{Ethical approval}

The study was approved by the Institutional Review Board of Seoul National University Bundang Hospital (IRB No. B-2001589-701) and performed in accordance with the principles of the Declaration of Helsinki. Written informed consent was obtained.

\section{Patient consent}

The patient provided written informed consent for the publica- tion and the use of his images.

\section{ORCID}

Joonho Lim https://orcid.org/0000-0002-4556-1536

Jeongseok Oh https://orcid.org/0000-0003-2001-084X

Seokchan Eun https://orcid.org/0000-0003-4372-6955

\section{REFERENCES}

1. Shokri T, Kadakia S, Saman M, Habal MB, Kohlert S, Sokoya M, et al. The paramedian forehead flap for nasal reconstruction: from antiquity to present. J Craniofac Surg 2019;30:330-3.

2. Angobaldo J, Marks M. Refinements in nasal reconstruction: the cross-paramedian forehead flap. Plast Reconstr Surg 2009; 123:87-93.

3. Chang SJ, Qiao C, Chang L, Gao W, Jin Y, Ma G, et al. A 7-year follow-up study on untreated deep or mixed facial infantile hemangioma in East-Asian patients: when propranolol was not yet an option. J Dermatol 2019;46:962-6.

4. Senturk E, Dagistanli N, Calim OF, Ozturan O. Nasal reconstruction following a dog bite. J Craniofac Surg 2019;30:22335.

5. Burget GC, Menick FJ. The subunit principle in nasal reconstruction. Plast Reconstr Surg 1985;76:239-47.

6. Bath K, Aggarwal S, Sharma V. Sushruta: father of plastic surgery in Benares. J Med Biogr 2019;27:2-3.

7. Climo MS. Nasolabial flap for alar defect: case report. Plast Reconstr Surg 1969;44:303-4.

8. Park CY, Chang H, Jeong EC, Lee YJ. A case of giant rhinophyma showing difficulty in nasal respiration. J Korean Soc Aesthetic Plast Surg 2008;14:165-9.

9. Krausz AE, Goldberg DJ, Ciocon DH, Tinklepaugh AJ. Procedural management of rhinophyma: a comprehensive review. J Cosmet Dermatol 2018;17:960-7. 